\title{
Tri-package White LEDs as a High-Color-Quality Lighting Source with Cd-free Quantum Dots
}

\author{
Ji Hye Oh, Keyong Nam Lee, Soyeon Yoon, Young Rag Do \\ Department of Applied Chemistry/Kookmin University \\ 77 Jeongneung-Ro, Seongbuk-Gu, Seoul, 02707, Korea \\ ohjh@kookmin.ac.kr; ykn21@kookmin.ac.kr; yoonso19@kookmin.ac.kr; yrdo@kookmin.ac.kr
}

\section{Extended Abstract}

In this study, we fabricated high-color-quality tri-package white-light-emitting diodes (WLEDs) using an InGaN blue LED and broadband-emitting green $(\mathrm{G})$ or red $(\mathrm{R})$ quantum dot (QD)-based down-converted LEDs (DC-LEDs) to realize high-color-quality WLEDs with various correlated color temperatures (CCTs) from cool white to warm white. To evaluate the color qualities, we adopted a color rendering index (CRI, Ra) and the TM-30-2015 color fidelity index (CFI, Rf), a color gamut index (CGI, Rg) and a color vector graph which is an improved version for color metrics to overcome the problems associated with the CRI [1,2]. The new color metrics of TM-30-2015 were developed by the Illuminating Engineering Society of North America (IES).

To fabricate monochromatic $\mathrm{G}$ and $\mathrm{R}$ down-converted LEDs, we synthesized Cd-free $\mathrm{G}$ and R QDs, broadband-emission I-III-VI2-based AgIn5S8/ZnS for G and CuInS2/ZnS for R by a colloidal hot-injection method. The AgIn5S8/ZnS and CuInS2 and ZnS QDs showed corresponding peak wavelengths of $514 \mathrm{~nm}$ and $589 \mathrm{~nm}$ and broadband full-width-at-halfmaximum (FWHM) values of $97 \mathrm{~nm}$ and $115 \mathrm{~nm}$ due to donor-acceptor recombinations [3]. They also showed photoluminescent quantum yields (PLQYs) of $75 \%$ and $85 \%$, respectively.

To realize tri-package WLEDs, we fabricated monochromatic G and R QD-based DC-LEDs using a long-wavelength pass-dichroic filter (LPDF), which reflects blue light to prevent color mixing from the excitation source of the blue LED and transmit G and R QD emission light [4]. To fabricate monochromatic G or R QD-based DC-LEDs, G and R QDs were mixed with Si-binder and put into an InGaN blue LED cup as an excitation source. Subsequently, LPDF was simply capped onto the LED cup to realize the monochromatic G or R QD-based DC-LEDs. The G and R QD-based DC-LEDs show peak wavelengths of $533 \mathrm{~nm}$ and $639 \mathrm{~nm}$ and luminous efficacy (LE) rates of $102 \mathrm{~lm} / \mathrm{W}$ and $42 \mathrm{~lm} / \mathrm{W}$, respectively. The peak wavelengths of the QD-based DC-LEDs were red-shifted due to the agglomeration of QDs which occurred while mixing them with the Si-binder [5].

Finally, the InGaN blue LEDs (peak-wavelength of $448 \mathrm{~nm}$, FWHM of $22 \mathrm{~nm}$ and LE of $18 \mathrm{~lm} / \mathrm{W}$ ) and G, R QD-based DC-LEDs were arranged in a triangular shape and applied separately with a total current of $180 \mathrm{~mA}$ at various CCTs of $10,000 \mathrm{~K}, 6,500 \mathrm{~K}, 5,000 \mathrm{~K}, 3,500 \mathrm{~K}$, and 2,700 K. When realizing cool to neutral white $(10,000 \mathrm{~K} \sim 5,000 \mathrm{~K})$, the portions of the applied current of the blue LED and the G QD-based DC-LEDs are higher than that of the R QD-based DC-LED. On the other hand, when realizing warm white $(3,500 \mathrm{~K}$ and 2,700 K), the portion of the applied current of the R QD-based DCLED is higher than that of the B LED or the G QD-based DC-LED. For this reason, the LEs of the WLEDs show relatively low values with warm white of $2,700 \mathrm{~K}(41 \mathrm{~lm} / \mathrm{W})$ compared to cool white at $10,000 \mathrm{~K}(52 \mathrm{~lm} / \mathrm{W})$ due to the low LE of the R QD-based DC-LED. The color qualities of WLEDs show high values (CRI: 86 94, CFI: 79 94 and CGI: 103 106) from $10,000 \mathrm{~K}$ to $2,700 \mathrm{~K}$. These results indicate that the tri-package WLED can be a good candidate for high-color-quality white lighting with various CCTs.

\section{References}

[1] D. A. P. T. Fini, K. W. Houser, Y. Ohno, M. P. Royer, K. A. G. Smet, M. Wei, L. Whitehead, "Development of the IES method for evaluating the color rendition of light sources," Opt. Express. vol. 23, pp. 15888-15906, 2015.

[2] M. P. Royer, "IES TM-30-15 Is Approved-Now What? Moving Forward with New Color Rendition Measures," Leukos, vol. 12, pp. 3-5, 2016. 
[3] N. S. Han, H. C. Yoon, S. Jeong, J. H. Oh, S. M. Park, Y. R. Do, and J. K. Song, "Origin of highly efficient photoluminescence in AgIn ${ }_{5} \mathrm{~S}_{8}$ nanoparticles," Nanoscale, vol. 9, pp. 10285-10291, 2017.

[4] J. R. Oh, S.-H. Cho, H. K. Park, J. H. Oh, Y.-H. Lee, Y. R. Do, "Full down-conversion of amber-emitting phosphorconverted light-emitting didoes with powder phosphor and a long-wave pass filter," Opt. Express. vol. 18, pp. 11063$11072,2010$.

[5] M. Ko, H. C. Yoon, H. Yoo, J. H. Oh, H. Yang, Y. R. Do, "Highly efficient green Zn-Ag-In-S/Zn-In-S/ZnS QDs by a strong exothermic reaction for down-converted green and tripackage white LEDs," Adv. Func. Mater., vol. 27, pp. 1602638, 2017. 